a positive result would be an indication for a thorough examination to exclude gonorrhoea.

It has always been a matter of surprise to me that medical officers of health whose chief concern is with the preventive aspect of medicine have not taken more advantage of the ophthalmia neonatorum notifications to trace untreated cases of gonorrhoea. About 50 per cent of cases are said to be due to the gonococcus but no financial or other stimulus is given to general practitioners to take smears or cultures (or smears and cultures) of the eye discharge in order that those due to the gonococcus can be identified. If a small fee were payable to doctors for doing this work the mothers of the gonococcal cases could be visited and be persuaded to attend a special clinic for examination.

Finally, I realize that there are a number of V.D. control measures which have been advocated from time to time and which I have not mentioned. I have done this not because these methods are of little importance but because I have had insufficient experience of their mode of operation to come to any decision on their value. I should like to add this however, that if we are ever to achieve adequate control of venereal disease now is the time for action when public interest in its prevalence has been awakened out of the lethargic state in which it has lain for the past twenty years.

\title{
REFERENCES
}

Berger, F. M. (1943) J. Path. Bact., 55, 363.

\section{DISCUSSION ON THE PRECEDING PAPERS}

Col. L. W. Harrison, who opened the discussion, said :

"I have asked to be allowed to speak early in this discussion because I believe that many people are not at all clear about the powers of the Ministry of Health in regard to appointments and arrangements generally under the V.D. scheme. At least $I$ judge this from the numbers of people who come to me asking me to give them appointments as V.D. officers. First of all, to dispel any impression which might be created by Dr. Price's remarks that our V.D. arrangements were the world's worst, I should like to say that I have visited clinics in a number of countries both on this side of the Atlantic and on the other and, by and large, I think that our clinics are as good as the others. I do not say that our best is the best in the world, or any nonsense of that kind, but the average of ours, with all their imperfections-and nobody is more conscious of the imperfections of our clinics than I am-is as good as the average elsewhere.

"Except in regard to Dr. Price's remarks about almoners-and I will leave that subject to others-I agree with much that has been said by the openers of this discussion but $I$ propose to show that we could not approach the ideal we want unless either: (a) hospital staffs, local authorities and all concerned with appointment of V.D.O.'s and with all other arrangements which go to the making of successful clinics, design of premises and so forth, are educated to the same pitch of keenness as ourselves; or $(b)$ the whole organization and administration of the V.D. scheme is centralized in the Ministry of Health.

"I will explain by examples. Prior to the coming into operation of the Local Government Act of 1929 (in April, 1930) the Ministry of Health exercised a fairly close supervision and medical officers of the Ministry made routine inspections of clinics. The inspections were necessarily infrequent because there were only three or four medical officers available for the work and I calculate that it would have taken one M.O. from two to three years to inspect all the clinics in this country if the visits were timed to coincide with sessions ; but they did keep us much more closely in touch with the work than after 1929. Appointments of medical officers were mostly in the hands of the staffs of the hospitals at which the clinics were situated and very often the medical officer appointed was a member of the staff, or someone who was persona grata with the staff, rather than the best person available in the area. The approval of the Ministry of Health was necessary but it was often difficult to find specific objections to a choice although local information might suggest that it was not the best that could have been made. Frequently hospitals refused to appoint any nominee of the local authority, although he might have been obviously the best man for the work and frequently when cogent criticisms were made of the medical officer's work, his colleagues supported him through thick and thin. Very loyal and all that but not making for efficiency. The fact is that very often hospital staffs do not regard V.D. work as requiring any special skill and experience. When I have said emphatically that for a given appointment the medical officer should be someone whose prime interest is venereal diseases, members of the hospital staff attending the conference for discussion of the arrangements of a clinic have exclaimed that surely I would not expect a medical officer to confine his interest to V.D. ; how narrowing! And so forth. They had not studied Osler on the subject of syphilis. The main point is that people who are not aware of the mental equipment necessary in a good V.D.O. are not the people to be entrusted with the choice of one. 
" In those years the voluntary hospitals had the whip hand because they could terminate the agreement for the conduct of the clinic in their premises and the local authorities concerned would then have had no premises to which to move the clinic. There is little wonder that local authorities had to give way on many points on which we felt that the best interests of the V.D. scheme were not being served.

"The Local Government Act of 1929 had some good effects and some bad ones. Amongst the good ones was the fact that it forced the medical officer of health, who might not hitherto have taken much interest in clinic arrangements, leaving all that to the Ministry, to look into the matter more closely. Further the local authorities became hospital authorities with greatly expanded resources in the form of premises and it became possible to remove clinics to hospitals under the control of the local authorities if this should seem to be advisable. Many voluntary hospitals who had hitherto adopted a take-it-or-leave-it attitude then began to discover that the V.D. clinics in their premises were definite assets.

"One bad point is the reduction of the Ministry's supervision and another is the fact that improvements of premises have to come out of the local authorities' block grants. This, I think, tended to make local authorities reluctant to introduce any improvements which we might suggest to them and often enough they would ask why we had not thought of it at the time the Ministry of Health paid 75 per cent of all expenditure by local authorities on the V.D. scheme. In spite of all these disadvantages it has to be admitted that very great improvements were effected in the service between 1916 and 1939.

"To take some of Dr. Price's points in detail :

"Premises. - You would be surprised to know the number of times all our efforts to stimulate local authorities and hospitals to improve the premises in which the V.D. work was done were nullified by the local V.D. officers, who said that the premises in which they were workingusually some rooms in an out-patient department-were quite satisfactory. The most heartbreaking part of the business of securing reforms in premises has been the delays. The time spent by committees and councils considering simple improvements is to my mind simply terrible, and is one of the strongest arguments for centralization of control. I should mention, however, that by 1939 about a quarter of the number of clinics had been set up in premises specially designed for the work and in a fairly large proportion of the others the turnover was so small as not to call for any change.

" Medical staff.-In 1939 we had working in the V.D. scheme 357 medical officers of whom only 63 were primarily V.D. specialists and only 44 of those were in charge of clinics. The remainder were part-time workers and sometimes very much part-time for the simple reason that there was insufficient work locally to keep a V.D. officer fully employed.

"The requirement that a V.D. officer must have had 130 hours' training came into being with the Local Government Act of 1929. It was then decreed that the requirement of the Ministry's approval of each appointment should cease but it was felt that some standard should be laid down. It was felt that if the standard were too severe it would be impossible to obtain candidates for a large number of the posts because of the smallness of these and the scarcity of local talent. There is no doubt that there has been a great scarcity of medical men with good reliable knowledge on this subject, a consequence of the very slight encouragement which seems to be given by medical schools to take up the subject. So we made conditions which we judged would result in the smaller posts being staffed by reasonably reliable men who could do the routine work of a clinic although they might not be able to give opinions on cases requiring a profound knowledge of first principles. For the bigger places there would be a number of candidates from whom the best could be chosen. If I had my way, every clinic would be run by a medical officer who had been thoroughly trained both in the laboratory and the clinic and had passed an examination such as Army specialists had to pass when I was in the Army. They had to be able to set up a microscope and its lighting arrangements almost from scratch and they had to do Wassermann tests on borderline specimens not because they were necessarily expected to do these tests in their routine work but so that they should know the limitations of these tests and generally be in full sympathy with the laboratory workers.

"I do not altogether agree with Dr. Price's strictures on the laboratory service. After all, the premises and the pathologist have had to be approved by the Ministry-at least the majority have since they were set up before 1930 - and as many here know, we have exercised some supervision through the comparisons of serum tests which were in operation from 1924 to 1939 and will, I hope, be renewed after the war. Also we have made big steps in the direction of standardization of reagents, the most notable being the central supply of reliable complement serum in liquid form. A decided blemish in the arrangements is the delegation of the testing by many approved pathologists to laboratory technicians. It is not easy to stop this practice when the approved pathologist is a learned professor who assures us that he is personally responsible for the tests, although anybody who has ever done serum tests knows that one can be responsible for the accuracy of such work only by sitting over the tester and watching his every movement; on those conditions one might just as well do the work oneself.

"At the beginning of this war I wanted the expansion centralized but it was ruled that we had no power to do it under present Regulations or that it was undesirable. If we had had centralization of the expansions we should have had experienced officers in charge of the additional treatment facilities ; they would have had to travel from place to place but that could no doubt have been arranged. Now I am afraid the opportunity has gone, the services having got all the good men we had available for staffing of new clinics in the earlier days of the war. Further the setting up of the new clinics would have been far speedier than it has been. I know nothing more 
calculated to make one a convinced totalitarian than the maddening delays of some local authorities over simple expansions. To secure centralization of control, reform of the method of appointment and the other improvements which we who know what is necessary for efficient management of venereal diseases desire, would require new legislation. It may be that, under a scheme for a comprehensive medical service, venereal diseases will receive more adequate consideration than in the past. In the meantime we must go on trying to educate the people who matter: they have a lot to learn."

Brig. T. E. Osmond (President) agreed with many of the assertions of the speaker, especially with regard to the poor accommodation in many of the clinics and to the inadequate equipment of some of the laboratories. He thought that Dr. Orpwood Price had taken an unduly narrow view when he had spoken of the benefit to patients ; surely the aim was the benefit to the whole community. He was entirely at variance with Dr. Orpwood Price on the subject of the lady almoner whose help could be of great value in the venereal diseases clinic; in fact Brig. Osmond was of opinion that a social service worker as well as an almoner was desirable. He was in agreement with the suggestion to form a national venereal diseases service because such a service would produce better results than could be attained at present, especially if more hospital beds became available for the treatment of patients. As regards prophylaxis, he considered that it might be ideal in theory but that there were very many difficulties in the way of its practical application. Dr. Burgess had mentioned that seventy-two persons in his area had been examined for venereal disease under Regulation 33B. Brig. Osmond thought it would be useful to know how many of these had been found to be suffering from a venereal disease.

Dr. Mary Michael-Shaw commented on the omission by the speakers of any mention of the position of the trained nurse whose knowledge on this subject was lamentably poor. She had few opportunities of acquiring knowledge and still fewer of obtaining any practical nursing experience ; in some hospitals there was antagonism to the attendance for training of junior nurses in the venereal diseases clinic.

The lady almoner in her opinion was indispensable to the efficient clinic. It was important to recognize that matrimonial difficulties did not constitute the greater part of the social work in the clinic. At the Royal Free Hospital the number of unmarried pregnant girls brought to the clinic this year was 474 , of whom 80 were found to have been infected. The amount of social work needed before and after the baby was born was very great. The provision of a secure background for these girls and sometimes of a little financial stability would do much to prevent many of them from drifting into prostitution. This was only one of the important aspects of the almoner's work. The dismissal of the almoner from the venereal diseases clinic would be a retrograde step, particularly so when the Interim Report on Social and Preventive Medicine of the Royal College of Physicians had only recently been published.

Sister E. Joyce said that in the past the nurse in training had received very little instruction in venereal diseases. Some slight theoretical teaching had been included in the syllabus but, unless she had nursed the occasional venereal patient in a general ward, her knowledge remained negligible. In general hospitals the V.D. clinic was isolated; the resultant secrecy had greatly discouraged the nurse's professional interest. Recently there had been a change in the national attitude toward these diseases and the public desired more knowledge ; greater knowledge was also demanded by the intelligent nurse and it could best be gained by practical work in the clinic.

It was Miss Joyce's experience as a sister-in-charge of a V.D. clinic that the nurses left the clinic grateful for the opportunity of sharing important work and in many instances wishing to continue it. There was an urgent need for nurses who had a broad outlook on life, an intelligent interest in social conditions and a sound knowledge of the diseases and their treatment. It appeared imperative that nurses should have specialized training so that there would be available a nucleus of nurses adequately trained to deal with the diseases and with the social problem.

Miss Joyce suggested that nurses in training should work in V.D. clinics and wards for one month to obtain a minimum of practical knowledge. This step had been tried in Guy's Hospital for the last year where the nurses worked in the clinic during their second training year; they acquired a good knowledge and a broader outlook. For State registered nurses there should be available a three months' course on the completion of which a certificate of proficiency should be issued. The industrial nurse also should have had some practical experience in a V.D. clinic and should have some knowledge of the infectivity of the diseases. It was surprising that no mention had been made of the need for V.D. knowledge and training in the articles on the industrial nurses' work recently published in the Nursing Times.

Dr. R. M. Warren, referring to education on venereal disease, thought that although much information was being given to the general public there was considerable variation in the teaching given to medical students and that there was a great need for uniformity in this teaching in order to enable medical men in the future to deal with the inquiries likely to follow the publicity campaign. Advantage had been taken of the publicity to circularize a warning to all general practitioners in his own area that syphilitic infection was becoming more frequent and to remind them of the facilities available at the clinic. As there appeared locally to be a wide variation in the treatment of gonorrhoea with a tendency to overcaution in dosage, the attention of the practitioners had been drawn to the latest recommendations of the Ministry of Health on the chemotherapy of gonorrhoea. The reminder had been well received.

The almoner played an important part in the services of the clinic. Her part could not be undertaken by the medical officer who usually had insufficient time to discuss at length the frequent domestic problems and little or no time to devote to the after-care of the increasing number of young girls. 
As regards the practical working of Regulation 33B the information he had been able to obtain for the identification of contacts had been distressingly vague.

Dr. Warren was strongly of the opinion that more efficient measures were urgently needed to compel the parents of a syphilitic child to undergo treatment.

Dr. Hamilton Wilkie expressed his preference for central control of the venereal diseases service ; possibly regional control was also advisable. Thus there would be some certainty of the senior V.D. officer being adequately experienced and qualified. In his opinion the senior officers should have laboratory experience as well as clinical experience. With regard to the work of special practitioners and the general practitioners who might treat venereal diseases, he thought that it should be made a general rule that the diagnoses be checked by the senior V.D. officer and that the treatment given be checked periodically.

In Leicester he had found that a few general practitioners treated cases entirely on their own lines and sometimes their diagnosis was incorrect. They had received two lectures from the senior V.D. officer which seemed to have effected some improvement. In the Leicester Royal Infirmary it was a rule that all nurses in training attend two lectures on venereal diseases ; when possible each nurse worked for three months in the V.D. ward during her training.

He thought that public lectures and propaganda in general on venereal diseases were very useful, but he felt that an impression was being created that Great Britain was rotten with venereal disease. This impression was incorrect as was also the unjust suggestion that the American troops were responsible for the increase in infection.

Mr. Hamish Nicol said that he considered the work of almoners to be invaluable especially in dealing with defaulters and with contacts. His experience of travelling patients indicated that more efficient cooperation between one clinic and another was urgently needed. The itinerant patient's treatment book (V.15) was sometimes irregularly retained at a clinic. Other complaints were that treatment had not been recorded and did not follow any system of dosage or of period of time, that injections had been repeated haphazardly and that the choice of remedy and dosage was unsuitable. Also, blood tests had been frequently neglected and clinical notes were invariably absent.

Commenting on the sanctimonious attitude of mind of the nursing profession toward venereal patients, he said a more compassionate outlook had long been overdue. He suggested that every senior nurse in training should work for three months in a V.D. clinic. Probationer nurses had been of little use in his clinic and were a potential danger to the patients and to themselves.

The present training of V.D. officers was unsatisfactory. Some medical officers had attended one of his clinics for a few sessions and had then applied to be appointed as specialists. He considered that a course of instruction of several months' duration followed by an examination should be designed. Medical officers who had not passed the test should not be appointed.

Dr. David Nabarro felt that further education was as necessary for the medical profession as it was for the general public. According to Dr. Percy Stocks (Lancet, 29th May, 1943) syphilis was registered as the cause of death in 2,790 cases during 1942 but a much larger number of cases of cardiovascular and nervous system degenerations were omitted because the syphilitic nature had not been registered on the death certificates. In consequence, morbidity due to Spirochaeta pallida has been greatly underestimated. Sir William Osler had stated his belief (1917) that syphilis caused a greater number of deaths than did tuberculosis. Dr. Nabarro believed this to be equally true today. The underestimation of the importance of venereal diseases by the medical profession could be corrected only by education, and it was a duty of this Society to provide education. He complained also of the lack of support and of cooperation by county councils and by medical officers of health. Although occasionally they succeeded in tracing defaulting patients, in general their interest was only slight. He agreed with Dr. Price that well-equipped laboratories and competent serologists were essential but he disagreed with Dr. Price's assessment of the value of almoners and social service workers who were doing good work. Parents who refused to bring their infected children for treatment could, he thought, be dealt with under the Children's Charter (1909), for refusal to obtain adequate treatment could be regarded as cruelty. There was a definite need for close cooperation between one clinic and another ; as regards treatment, a generally accepted scheme of treatment and dosage would be of great value. This scheme should be brought about by our Society.

Sqni. ldr. J. V. MacGregor thought that there seemed to be general agreement that the present venereal diseases scheme should undergo reconstruction. He agreed that the Society should appoint a committee to consider possible improvements including those which might require new legislation. Regulation 33B had its disadvantages. The first notifications of sources of infection were usually buried in a card index and he understood that for a very small proportion only of first notifications had a second notification been received.

Dr. R. Forgan said that implicit in the title of the discussion was the suggestion that those engaged in the treatment of venereal diseases had not been receiving a square deal and that for this'political influences seemed to be responsible. There were grounds for questioning the sincerity of the intentions of a Government which, through its Ministry of Health, launched a campaign of popular education on venereal diseases while its Ministry of Labour treated prostitution as a reserved occupation and while solicitation on the streets flourished under the protection of the Ministry of "Home Security."

There was one fact of the treatment of gonorrhoea to which reference had not been made, namely, that less than half the number of patients with gonorrhoea in England were coming to clinics for treatment. Who was treating the majority? Was there a black market for the sul- 
phonamide drugs? Probably many cases were being treated by private-practitioners and this must be borne in mind in preparing plans for the more effective control of venereal diseases.

Dr. W. N. Mascall supported the proposal of a national V.D. scheme. Formerly when he had been an assistant medical officer of health his duties had included those of V.D. officer, maternity officer to a municipal maternity home, child welfare officer, smallpox officer and meat inspector. Under this unsatisfactory arrangement it had not been uncommon that when he was settled at the V.D. clinic an urgent call away to a maternity case had ended the clinic for that evening ; the V.D. work had always seemed to be at a disadvantage in comparison with the other services. A V.D. officer should be a full-time V.D. officer and not a jack-of-all-trades.

With regard to almoners, he had worked in a hospital where there was an almoner, a health visitor who acted for both T.B. and V.D. and a trained clerical staff. The activities of the health visitor had had to be curtailed because people saw her and thought that the person called upon was suffering from T.B. whereas the T.B. patients thought that they would be considered to be suffering from V.D. Thus the patients were upset and defaulted. A trained almoner was unnecessary in a clinic provided there was a good trained clerical staff.

The London County Council were conducting a well-advertised training scheme for nurses which was meeting with a certain amount of response. It was a four-months' course comprising both in-patient and out-patient experience and led finally to some form of proficiency certificate.

Much had been said about the 130 hours of training of medical officers. This had always been unsatisfactory but in war-time it should not be criticized too much because there was a definite shortage of medical personnel. Some of the people had worked during the 130 hours but some had not and would never make good officers. After the war considerable weeding out would be required and Dr. Mascall agreed with Dr. Burgess that a diploma or some form of examination should be instituted and a definitely higher standard of efficiency demanded.

Dr. A. E. W. McLachlan drew attention to the inadequacy of the Local Government Board qualification period of 130 hours' experience for V.D. medical officers. He knew of no other speciality for which so brief a course was required and he suggested that an officially prescribed syllabus of postgraduate instruction with a subsequent clinical and practical examination was desirable. He also referred to the ill-defined position of V.D. male nursing orderlies who, although highly skilled, were not on the State Register. He was of the opinion that their status should receive some official recognition.

It was well known that the treatment book of travelling patients revealed considerable inequality in the standards of investigation and treatment. The situation might be improved by the setting up of authoritative standards.

Amongst other suggestions he put forward were (1) that regionalization of V.D. services should be made possible, (2) that funds for research should be made available, and (3) that standardized reagents for laboratory tests should be obtainable from a central research laboratory.

Dr. McLachlan thought that there were various opinions on the duties of an almoner in relation to contact tracing. Some almoners considered that this special duty was not in their sphere of action. If contact tracing was to be an integral part of the future policy of the clinics it might lead to a duplication of social workers.

Dr. F. R. Curtis disagreed strongly with the adverse criticism by Dr. Price of the value of almoners in the clinics for this criticism was based on an entirely inadequate experience of their services. The opinion of this meeting was against Dr. Price's contention with the exception of the opinion of Dr. Mascall who was Director of the clinic where Dr. Price was the pathologist and where almoners were not employed.

Dr. R. Marinkovitch was glad to hear the position of the lady almoner thoroughly discussed. Many people had not any clear idea of the function of an almoner. At a meeting of the Society of the Medical Officers of Health he was asked to explain the nature of an almoner's duties in the clinic. It was evident from the Ministry of Health circular that some clerical work could be undertaken by the almoner. He was glad to state that an almoner had been appointed to his clinic to begin her work in January, 1944. On the introduction of an almoner to a clinic he feared that there might be some jealousy on the part of the nursing and of the clerical staffs. In order to do her best work the almoner should have an office to herself. Some medical officers of health were trying to delegate their duties arising from Regulation 33B to the V.D. officer who, in this event, would be only too glad to have the assistance of a lady almoner. The ideal position of a venereal diseases clinic was as a department of a general hospital but from some aspects it seemed easier to work in an independent clinic which, however, should be closely associated with a hospital or with other clinics and with adequate laboratory facilities.

Col. L. W. Harrison said that reference had been made to the relatively poor results of 33B in respect of two forms being received. But the advantage of 33B did not end there; it had been a means of informing medical officers of health about numbers of suspicious cases as sources of infection which they had never known of before. Medical officers of health had been encour: aged to take action on receipt of the first form which was in the way of persuasion to attend for examination. A number of medical officers of health had however been advised by their clerks that they were running grave risks. This was not so. All they had to do was to make the approach in a tactful way in the same manner as the American nurses were approaching known contacts.

He was sorry that Dr. Forgan had gone as he would have liked to have answered him on the question of prostitution and the reserved occupation. There had been a good deal of hard and shrewd discussion on this point and if anybody could suggest a way through the difficulties the suggestion would be welcomed. Every means of dealing with this problem had been gone into 
by the Minister of Health, the Home Office and Scotland Yard. What was to be done with these women? If they were put into the Services no one would associate with them and they could not be put behind barbed wire.

Another point was that the transference of documents was not being made out properly, especially for merchant seamen. A tremendous amount of work had been done to induce people to fill in these forms but the fact was that medical officers had seemed to hate putting pen to paper. For years he had worked for a standard of treatment for merchant seamen. He had had courses laid down; special cards had been issued; the League of Nations had recommended courses but this seemed to have had little effect.

Dr. Orpwood Price, in reply, thanked Col. Harrison for his talk but thought that the real trouble was money. There was not sufficient money to attract enough good candidates for this branch of Medicine.

As far as almoners were concerned he seemed to have brought a hornet's nest about his ears. If Dr. Curtis really had thought his remarks should not be published he (Dr. Curtis) would not have made such an onslaught on him. He thought his remarks should be published if only in order to obtain another point of view.

He thought that the Society, as suggested in his paper, should form a subcommittee to consider what should be done regarding a new deal for venereal diseases.

Dr. Burgess, also in reply, said that Col. Harrison referred to the fact that if the standard of V.D. training was raised to too high a level there would be great difficulty in filling all the appointments. His reply would be that if the scale of remuneration was raised the difficulty would be overcome.

Brig. Osmond had asked about the seventy-two persons who had attended voluntarily at the clinics. These were people concerning whom there had been one notification but they were not compulsory cases. He had had only five compulsory cases in the West Riding. The seventy-two had attended as the result of persuasive methods and he was unable to say how many were actually suffering from syphilis or gonorrhoea. Medical officers in charge of clinics were under no obligation to give this information to local authorities; they were compelled to give information on compulsory cases only.

With regard to the 130 hours' training the difficulty had been that local authorities had had the impression that a doctor who had spent 130 hours training in a large clinic automatically became a V.D. specialist ; it was something which had been bound to arise when the minimum time standard had been fixed at $\mathbf{1 3 0}$ hours. It was an impression which should be corrected. He agreed that nurses should have theoretical and practical training in venereology; either this Society or the Ministry of Health could stimulate the General Nursing Council to make venereal disease a compulsory subject in the State Registration examination.

With regard to the black marketing of the sulphonamide drugs he had known a clinic where the director had complained that his supplies of sulphathiazole were vanishing unaccountably. Thereupon he had set a trap and had found that an orderly had been taking the tablets and had been selling them to patients in the intermediate treatment department.

Col. Harrison had made it clear that persuasive methods did not lay local authorities open to actions for libel in cases under Regulation 33B. He wondered if the Ministry of Health could make this absolutely clear to town clerks because in the West Riding, although the administrative county took steps to persuade persons about whom there had been a single notification to attend a clinic, some of the county boroughs, of which there were eleven, did not. They just filed away the forms until the second notification turned up.

\section{Canadian control of venereal disease}

A national programme for the control of venereal diseases has recently been planned in Canada. The six measures adopted are the following. (1) Healthy, dignified education about venereal diseases. (2) Adequate facilities for the diagnosis and treatment of all patients having these diseases. (3) Charlatan and quack treatment of venereal disease to be suppressed. (4) Early and suitable prenatal care, including blood tests, for expectant mothers. This will help to prevent their babies being infected with venereal diseases. (5) General examination, with blood tests for syphilis, before marriage. (6) Effective measures for dealing with individuals and communities responsible for spreading these diseases. The National Defence Department and the Departments of Pensions and National Health and of Provincial Health are cooperating. Administrative machinery common to the Department of National Defence and the Federal Health Department has been set up. The Armed Forces through their respective medical services are providing up-to-date facilities for the prevention and cure of venereal diseases.-Venereal Disease Information, Washington, October, 1943.

\section{Statistics of cases reported under Regulation 33B}

Persons who have been found to be sources of venereal infection and who have been reported by at least two individuals undergoing treatment total 3,344 up to 30th September, 1943; of these two men and 150 women have undergone treatment. In addition, some 543 individuals out of 3,116 about whom only one report was sent to medical officers of health were persuaded, after an informal approach, to be examined and to undertake treatment.-_Hansard, 4th February, $1944,1529$. 